\title{
Morfología y anatomía de órganos vegetativos de Tropaeolum incisum [Tropaeolaceae]
}

\author{
Morphology and Antomy of Vegetative Organs of Tropaeolum \\ incisum (Tropaeolaceae)
}

\author{
Bulacio Eva1*; María I. Mercado²; Graciela I. Ponessa ${ }^{2}$ \\ 1 Laboratorio de Taxonomía Fanerogámica. \\ 2 Instituto de Morfología Vegetal. \\ Fund. M. Lillo, Miguel Lillo 251, (T4000JFE) San Miguel de Tucumán, Argentina. \\ * Autor corresponsal: ebulacio@lillo.org.ar
}

\begin{abstract}
- Resumen - Bulacio Eva; María I. Mercado; Graciela I. Ponessa. 2017. "Morfología y anatomía de los órganos vegetativos de Tropaeolum incisum (Tropaeolaceae)". Lilloa 54 (2). Se estudió la anatomía de los órganos vegetativos de $T$. incisum, especie que crece en el centrooeste de la Argentina y este patagónico. El material colectado fue fijado, diafanizado, cortado con micrótomo rotativo de Minot y posteriormente teñido. Se observaron rasgos anatómicos de valor adaptativo: presencia de tricomas secretores unicelulares y papilas en la epidermis de rizomas aéreos, cristales en las células del mesofilo y papilas en los márgenes foliares.

Palabras clave: Adaptación; Tropaeolaceae; Tropaeolum incisum; Patagonia.
\end{abstract}

> Abstract - Bulacio Eva; María I. Mercado; Graciela I. Ponessa. 2017. "Morphology and anatomy of vegetative organs of Tropaeolum incisum (Tropaeolaceae)". Lilloa 54 (2). The anatomy of the vegetative organs of $T$. incisum was studied. This species grows in the Center-west of Argentina and Patagonian east. The collected material was fixed, cleared, cut with a Minot rotary microtome. Anatomic features of adaptive value, presence of unicellular secretory trichomes and papillae in the epidermis of aerial rhizomes, crystals in mesophyll cells and papillae at leaf margins, were observed.

Keywords: Adaptation; Tropaeolaceae; Tropaeolum incisum; Patagonia.

\section{INTRODUCCIÓN}

Tropaeolum L. (Tropaeolaceae) es un género americano, de distribución andina, con más de 100 especies que habitan desde Méjico hasta el sur de Argentina y Chile (Andersson y Andersson, 2000). En la Argentina se reconocen 15 especies que habitan en tres regiones bien definidas: noroeste, noreste y centro-sur (Bulacio, 2015). Se caracterizan por la morfología de la corola zigomorfa, generalmente con lóbulos fimbriados o a veces enteros y por la presencia de un espolón nectarífero variable en forma, tamaño y color.

El género reviste importancia económica, ya que comprende a la «mashua» (Tropaeolum tuberosum Ruiz \& Pav. ssp. tuberosum)

Recibido: 24/04/17 - Aceptado: 12/09/17 cuyos tubérculos son cultivados, junto a la «oca» (Oxalis tuberosa Molina) y el «ulluco» (Ullucus tuberosus Caldas) como un recurso alternativo en la alimentación de los pobladores del altiplano (Fernández, 1973; Brücher, 1989; Hermann, 1992). Otras especies como T. pentaphyllum Lam. ssp. pentaphyllum y T. polyphyllum Cav., son utilizadas como ornamentales (Bulacio, 2015). Por su parte, en medicina popular T. majus L. es utilizada para el tratamiento de desórdenes cardiovasculares, infecciones del tracto urinario, asma y constipación (Gasparotto et al., 2009).

Tropaeolum incisum (Speg.) Sparre, es una de las 7 especies patagónicas comprendidas en la Sección Chilensia Sparre (Andersson y Andersson, 2000), que están adaptadas a climas semi-áridos. Se distribuye en Chile central y la Argentina, en las 
provincias de Mendoza, Neuquén, Chubut y Río Negro (Bulacio, 2015). Son hierbas robustas, crasas, apoyantes o postradas, con rizomas blanquecinos del que nacen tubérculos alargados (Fig. 1 E-F). Sus flores son solitarias, amarillas, rojizas, rosadas plenas o con máculas purpúreas, con un cáliz verde amarillento, bilabiado terminado en un espolón cónico y aleznado (Fig. 1 A-C). Crece en áreas semi-áridas al pie de los primeros contrafuertes cordilleranos, entre los 1000 y $3000 \mathrm{~m}$ snm, en estepas, arbustales bajos, sobre suelos sueltos y pedregosos hasta las áreas costeras del sur del país.

Se estima que en zonas áridas y semi-áridas, como resultado de un proceso evolutivo, han surgido especies que están adaptadas a condiciones extremas dando lugar a las plantas conocidas como xerófitas. Según Dickinson (2000) las plantas presentan adaptaciones morfológicas que se manifiestan principalmente en las hojas siendo considerado este órgano como el más variable e indicador de diferentes condiciones ambientales. Sin embargo, a veces no todas las características visibles proporcionan evidencia de su adaptación a la aridez y en ocasiones, tales cambios se observan a nivel tisular (Oppenheimer, 1960).

Metcalfe y Chalk (1950) estudiaron T. majus, T. aduncum Sm., T. peregrinum L. y T. pentaphyllum, y las caracterizaron por la presencia de células con mirosina, estomas anomocíticos en general en la superficie abaxial, ausencia de tricomas y tejido mecánico. Además, se realizaron estudios morfo-anatómicos de órganos vegetativos de T. majus (Zanetti et al., 2004), T. tuberosum ssp. silvestre Sparre (Bulacio y Ponessa, 2012), T. capillare Buchenau (Bulacio et al., 2013) y de las especies brasileñas, T. warmingianum Rohrb., T. brasiliense Casar. y T. sanctae-catharinae Sparre (Fromm-Trinta y Gonçalves-Costa, 1976).

En lo que respecta a las especies de la sección Chilensia sólo se citan estudios sobre los aspectos anatómicos y de la biología reproductiva de T. pentaphyllum (Fabbri y Valla, 1998) y algunos caracteres anatómicos foliares de T. incisum (Pyykkö, 1966).
En relación a los escasos antecedentes anatómicos de las especies de la Sección Chilensia, el objetivo del presente trabajo fue estudiar la morfología y anatomía de los órganos vegetativos de T. incisum a fin de identificar caracteres diagnósticos que indiquen su posible adaptación a condiciones de semi-aridez y que permitan diferenciarla del resto de las especies.

\section{MATERIALES Y MÉTODOS}

Se trabajó con material recolectado en áreas de la pre-cordillera de Neuquén y material de herbario. Parte del material fue herborizado y depositado en el Herbario de la Fundación Miguel Lillo (LIL). Se seleccionaron tres ejemplares al azar y de cada uno se tomaron muestras de sus órganos vegetativos.

\section{MORFOLOGÍA Y MICROSCOPÍA}

El material recolectado fue fijado en FAA (ácido acético, formol, agua, alcohol etílico 1:2:7:10) mientras que el material de herbario fue hidratado para su posterior procesamiento. Se realizaron cortes histológicos de $20 \mu \mathrm{m}$ de espesor, con micrótomo rotativo Minot, los que fueron decolorados en una solución de hipoclorito de $\mathrm{Na}$ al 50\%, lavados, teñidos con tinción doble sucesiva, Azul Astra-Safranina y posteriormente montados en agua: glicerina 1:1 (Zarlavsky, 2014).

Segmentos del tubérculo fueron deshidratados, incluidos en parafina, seccionados (10 $\mu \mathrm{m}$ de espesor) con micrótomo rotativo Minot y posteriormente coloreados con tinción doble sucesiva Safranina-Fast Green (Zarlavsky, 2014).

Para los estudios de venación se realizaron diafanizados empleando la técnica de Dizeo de Stritmatter (1973). La descripción de la arquitectura y venación foliar se realizó según la terminología de Hickey (1974) y Ellis et al. (2009). Para la clasificación de los estomas se usó la terminología propuesta por Dilcher (1974).

Los preparados se observaron en un microscopio óptico de luz compuesta-polarizada Karl Zeiss Axiolab con una cámara Zeiss 
Axiocam ERc 5s adosada. Las escalas fueron calculadas con software AxioVision Rel.4.8.

\section{MATERIAL ESTUDIADO}

ARGENTINA. Prov. Neuquén, Dpto. Aluminé., Ruta 23 orilla este del río Aluminé, 15-I-1982, Mallo 18155 (LIL). Dpto. Minas, camino a las lagunas de Epulafquén, bordeando el río Nahueve, 3658'40"S 7052'04”W, 1350 m snm, 9-I-2010. Bulacio y Ayarde 1311 (LIL); Reserva Epulafquén,

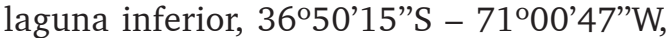
$1490 \mathrm{~m}$ snm, 10-I-2010. Bulacio y Ayarde 1333 (LIL).

\section{RESULTADOS}

MORFOLOGÍA

Y ARQUITECTURA FOLIAR

Tropaeolum incisum posee hojas basales simples y apicales compuestas, crasas, verde-grisáceas, glabras, de disposición alterna (Fig. 1 D y 2 A).
Las hojas simples, son peltadas, largamente pecioladas (Fig. 2 A); pecíolo de 70$100 \times 1-2 \mathrm{~mm}$, a veces helicoidales en la parte basal (Fig. 2 B). Lámina suborbicular, de aproximadamente $3-4 \mathrm{~cm}$ de diámetro, palmatisecta, dividida casi hasta el nervio medio en 7 a 9 lóbulos, en ocasiones plegada en su parte media, ápice obtuso y base decurrente (Fig. $2 \mathrm{C}$ ). La venación es palmada, actinódroma basal, con 8-10 venas de primer orden, de recorrido curvo hacia los lóbulos (Fig. $3 \mathrm{~A}$ ).

Las hojas palmati-compuestas presentan pecíolos de 10-60 x 2-3 mm (Fig. 2 A); la yema axilar de las mismas denota el punto de inserción al tallo (Fig. 2 D); son tetrafolioladas, en ocasiones los folíolos se dividen nuevamente en forma dicotómica, originando peciólulos de 3-5 mm de largo (Fig. 2 E). Cada folíolo es suborbicular, de 10-50 x 2,3$50 \mathrm{~mm}$, ápice obtuso y base decurrente (Fig. 2 F). En los folíolos de la hoja compuesta el número de venas primarias se reduce a
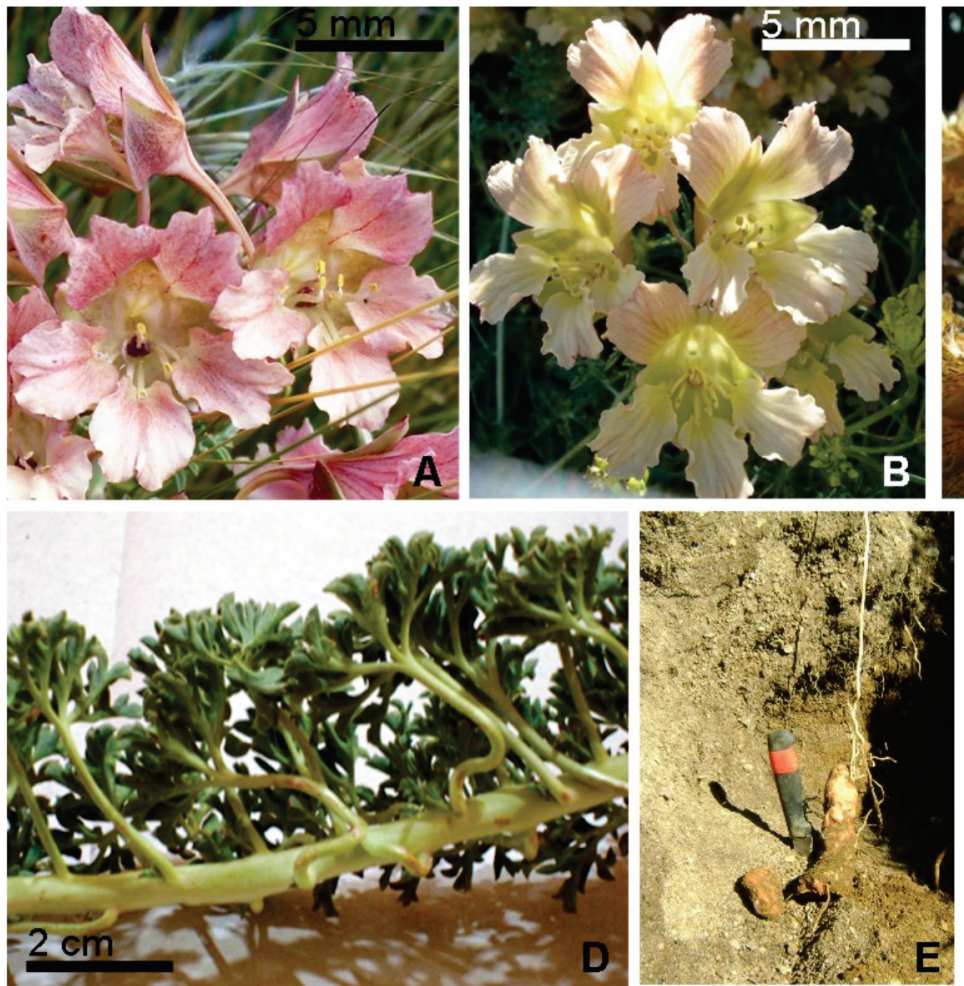
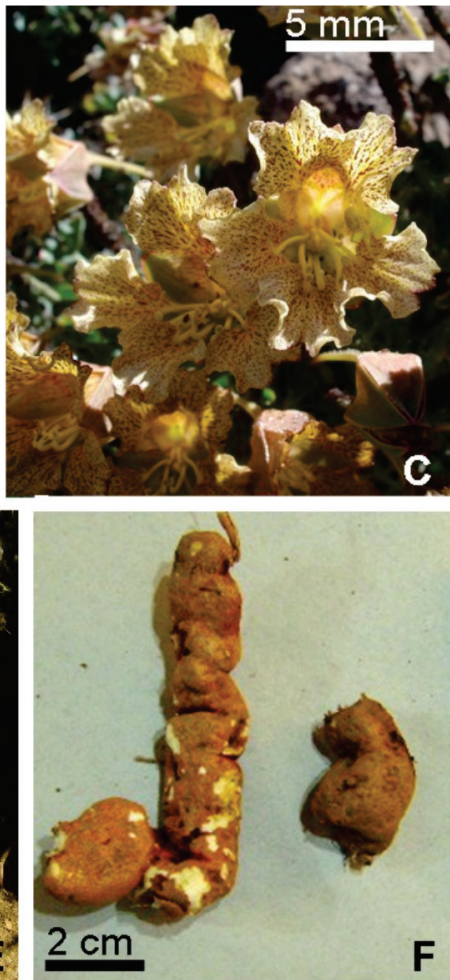

Fig. 1. Tropaeolum incisum. A-C) Flores (mostrando las diferentes tonalidades) D) Aspecto de una rama. E-F] Tubérculo. 
2-3 (Fig. 3 B). Las venas secundarias presentan las mismas características tanto en hojas simples como compuestas, cladódromas, alternas, decurrentes e irregularmente distribuidas. Éstas conforman con el nervio medio ángulos agudos moderados a anchos $\left(45-80^{\circ}\right)$, dividiéndose dicotómicamente en secundarias menores semi-craspedódromas hacia la región exmedial. En hojas palmati- sectas, las venas secundarias interiores comunican lóbulos adyacentes (Fig. $3 \mathrm{C}$ ).

Las venas terciarias divergen formando ángulos rectos a obtusos y presentan un curso reticulado ortogonal formando aréolas epimediales, uniendo áreas intercostales y exteriores (Fig. $3 \mathrm{C}$ ).

Las venas cuaternarias, correspondientes a la venación de menor orden, forman

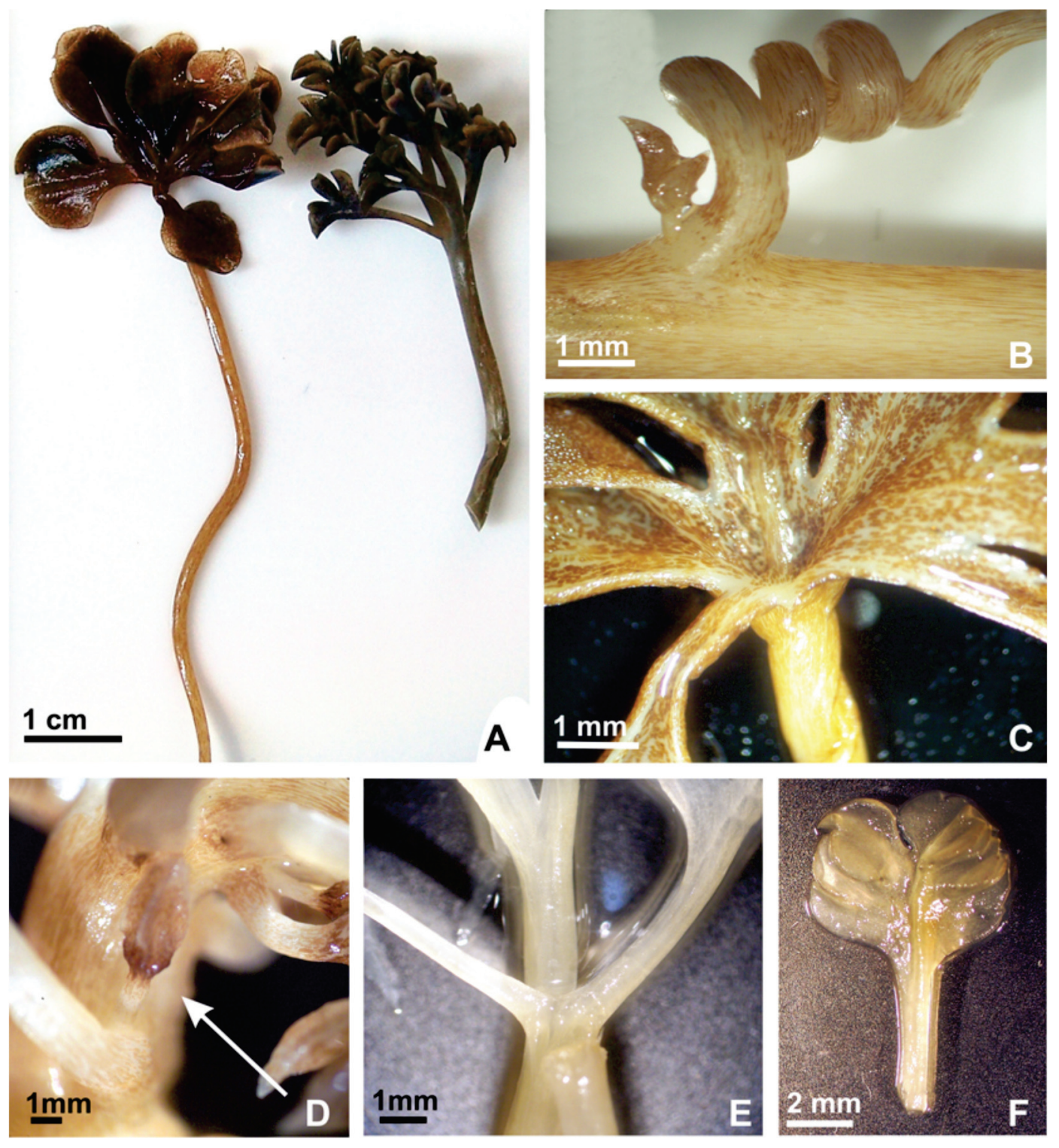

Fig. 2. Tropaeolum incisum, morfología. A) Dimorfismo foliar. Hoja simple palmatisecta profundamente lobada y palmati-compuesta. B] Pecíolo voluble. C) Detalle de la inserción del pecíolo, hoja simple palmatisecta. D, E, F] Hojas palmati-compuestas: D) Yema axilar (flecha]. E) Peciólulos. F) Folíolo suborbicular. 

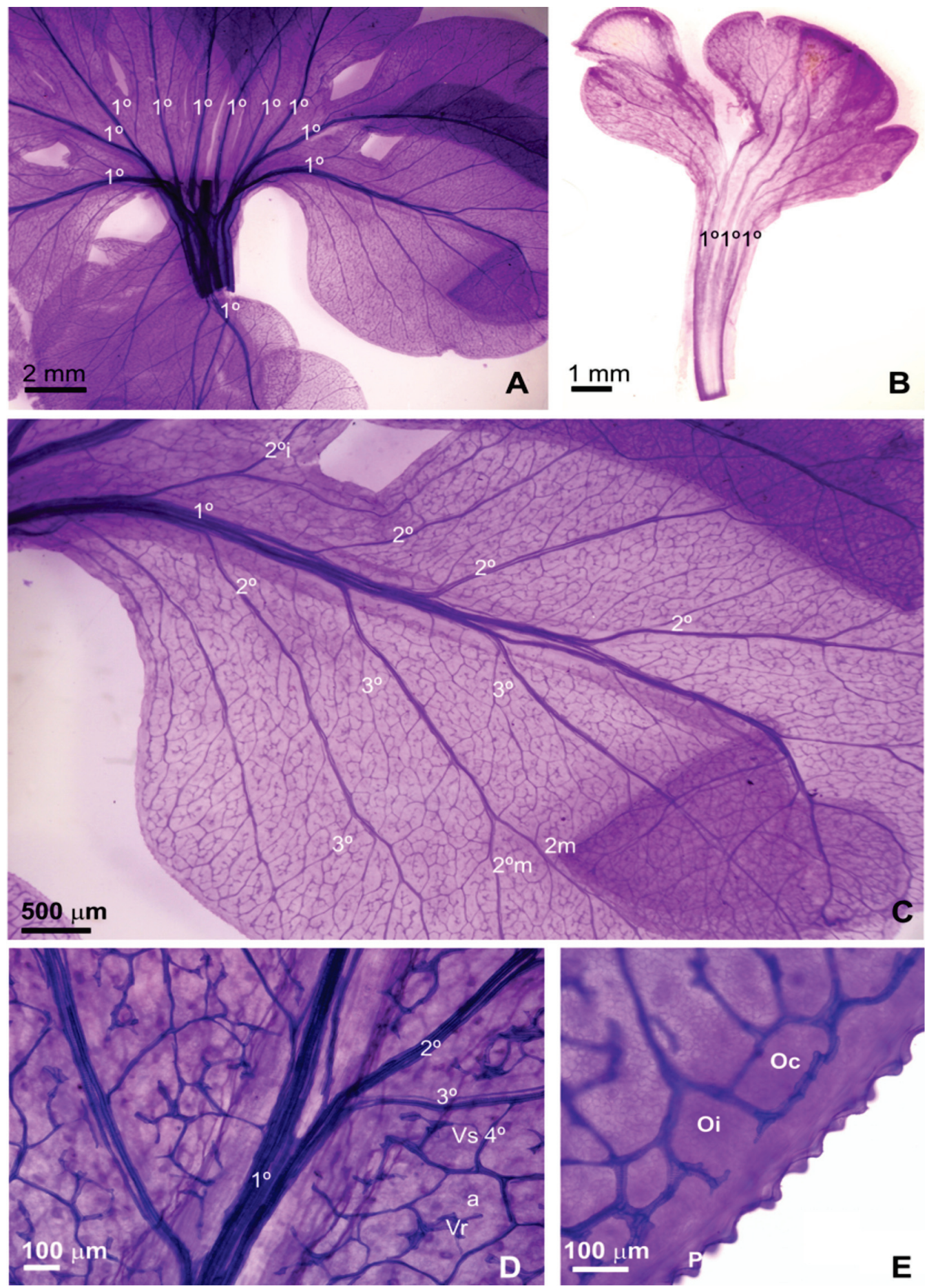

Fig. 3. Tropaeolum incisum, arquitectura foliar. A) Hoja simple, detalle de la venación palmada actinódroma basal. B] Hoja compuesta, venas primarias de aspecto flabelado. C) Venas secundarias y terciarias. D) Venas cuaternarias y aréolas. E) Margen papiloso. Referencias: 1을 venas primarias; $2^{\circ}$, venas secundarias; $3^{\circ}$, venas terciarias; $4^{\circ}$, venas cuaternarias; $2^{\circ} \mathrm{m}$, venas secundarias menores; $\mathfrak{L}^{0} \mathfrak{i}$, venas secundarias interiores; $a$, aréola; Vs, vénula simple; $\mathrm{Vr}$, vénula ramificada; Oc, ojal completo; Oi, ojal incompleto. 
aréolas poligonales dispuestas al azar con vénulas simples y ramificadas (Fig. 3 D). La venación última marginal es ojalada completa e incompleta. El margen se presenta liso a papiloso en su tercio terminal con series de 3-4 papilas superpuestas (Fig. $3 \mathrm{E}$ ).

\section{ANATOMÍA DE HOJA}

Las hojas, tanto simples como compuestas, presentan idéntica estructura a nivel anatómico. En vista paradermal, ambas epidermis poseen células poligonales de paredes anticlinales ligeramente lobuladas (Fig. $4 \mathrm{~A}$ y B). Hacia la región basal de la hoja suele
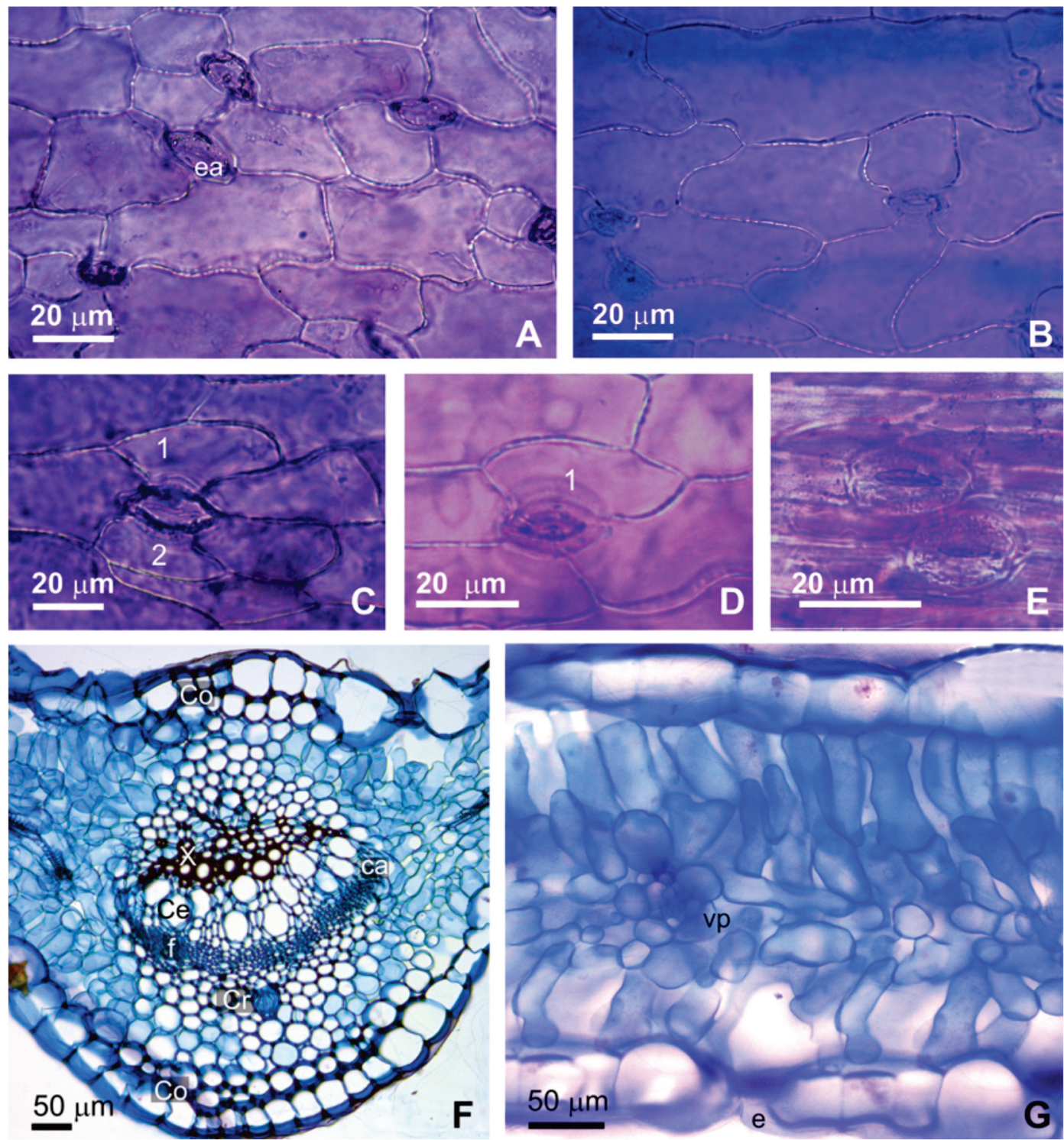

Fig. 4. Tropaeolum incisum, anatomía foliar. A) Epidermis adaxial. B] Epidermis abaxial. C] Aparato estomático braquiparacítico. D) Aparato estomático hemiparacítico. E) Estomas gemelos. FJ Transcorte a nivel de nervadura de 1 er. orden. G) Sección transversal de la lámina a nivel de nervio $2^{\text {rio }}$. Referencias: Ca, haz colateral abierto; Ce, canal esquizógeno; Co, colénquima; Cr, cristal; e, estoma; ea, estoma anomocítico; f, floema; vp, vaina parenquimática; $\mathrm{X}$, xilema; 1,2 , células acompañantes. 
predominar el eje longitudinal. Las hojas son anfiestomáticas con estomas anomocíticos y aparatos estomáticos braquiparacíticos (Fig. 4 C) y hemibraquiparacíticos (Fig. 4 D), raramente se observan estomas gemelos (Fig. 4 E), las células oclusivas miden $24( \pm 3,7) \mathrm{x}$ $13,8( \pm 1,9) \mu \mathrm{m}$. Presentan 134,28( $\pm 9,5) \mathrm{y}$ $95,7( \pm 8,7)$ estomas $\mathrm{x} \mathrm{mm} \mathrm{mm}^{2}$ en la epidermis adaxial y abaxial respectivamente. Se observan papilas, una por célula, restringidas al tercio superior del margen foliar.

En transcorte a nivel de las venas de primer orden, se observa la epidermis unistrata, 1-2 estratos de colénquima laminar subepidérmico, parénquima con cristales romboidales de oxalato de calcio, un único haz vascular colateral con conductos esquizógenos entre el xilema y el floema (Fig. 4 F).

En sección transversal, la lámina es isolateral, anfiestomática de 279,05 ( \pm 4,5) $\mu \mathrm{m}$ de espesor. Se observa cutícula delgada, epidermis unistrata de 54,1 $( \pm 12,2) \mu \mathrm{m}$ y 40,64 ( $\pm 10,7) \mu \mathrm{m}$ de espesor en la epidermis adaxial y abaxial respectivamente. Estomas ligeramente hundidos respecto a la superficie epidérmica. Parénquima en empalizada bistrato hacia la superficie adaxial de $81,9( \pm 12,7) \mu \mathrm{m}, 2-3$ estratos de tejido esponjoso, compacto de 40,3 $( \pm 5,3) \mu \mathrm{m}$ y parénquima en empalizada abaxial, unistrato, laxo de 46,59 $( \pm 6,2) \mu \mathrm{m}$. Los haces vasculares de menor orden se presentan colaterales cerrados, con vaina parenquimática continua (Fig. $4 \mathrm{G}$ ).

\section{ANATOMÍA DE PECIÓLULO}

La epidermis presenta células cuadrangulares de paredes anticlinales rectas. Se observan estomas anomocíticos y braquiparacíticos (Fig. 5 A). En sección transversal es subcircular. La epidermis es unistrata, con cutícula delgada, colénquima subepidérmico angular unistrato, 7-8 estratos de parénquima, un único haz vascular colateral de características similares a las del nervio medio foliar, rodeado por un anillo continuo de 1-3 estratos de células esclerenquimáticas. El peciólulo, cuando se divide nuevamente para dar lugar a un foliólulo, presenta haces me- nores, de iguales características anatómicas (Fig. 5 B).

\section{ANATOMÍA DE PECÍOLO}

Semejante en hojas simples y compuestas. En vista paradermal, las células epidérmicas y aparatos estomáticos presentan iguales características que las mencionadas anteriormente para el peciólulo de las hojas compuestas.

En sección transversal la región proximal y media presentan aspecto circular. Se observa cutícula delgada, epidermis unistrata, colénquima laminar subepidérmico uni a biestrato y 5-6 estratos de células parénquimaticas. Un anillo de 3-4 estratos de esclerénquima rodea el sistema vascular conformado por 5-6 haces vasculares colaterales abiertos de iguales características a los descriptos para la hoja, los mismos se disponen de forma circular dejando en su interior la médula parenquimática con células de mayor tamaño que las observadas por fuera del anillo esclerenquimático (Fig. 5 C). Los sectores donde el pecíolo se presenta voluble helicoidal, mantiene la estructura descripta, excepto a nivel del anillo esclerenquimático, el cual se observa adelgazado o interrumpido (Fig. 5 E).

La sección distal del pecíolo presenta aspecto semicircular con su cara adaxial plana. De estructura similar a las secciones media y proximal, se diferencia en su sistema vascular, el cual está formado por 5-6 haces vasculares (similares a los mencionados anteriormente) tres de ellos distribuidos en arco hacia la superficie adaxial y dos invertidos (Fig. 5 D).

\section{ANATOMÍA DE TALLO}

En vista paradermal, el tallo de estructura primaria presenta una epidermis de células cuadrangulares de paredes anticlinales rectas, aparato estomático braquiparacítico y hemibraquiparacítico, tricomas secretores unicelulares de extremo redondeado y papilas una por célula, distribuidas en forma irregular (Fig. 6 A). En sección transversal presenta aspecto circular de 2-3 mm de diámetro, epidermis unistrata de células de as- 

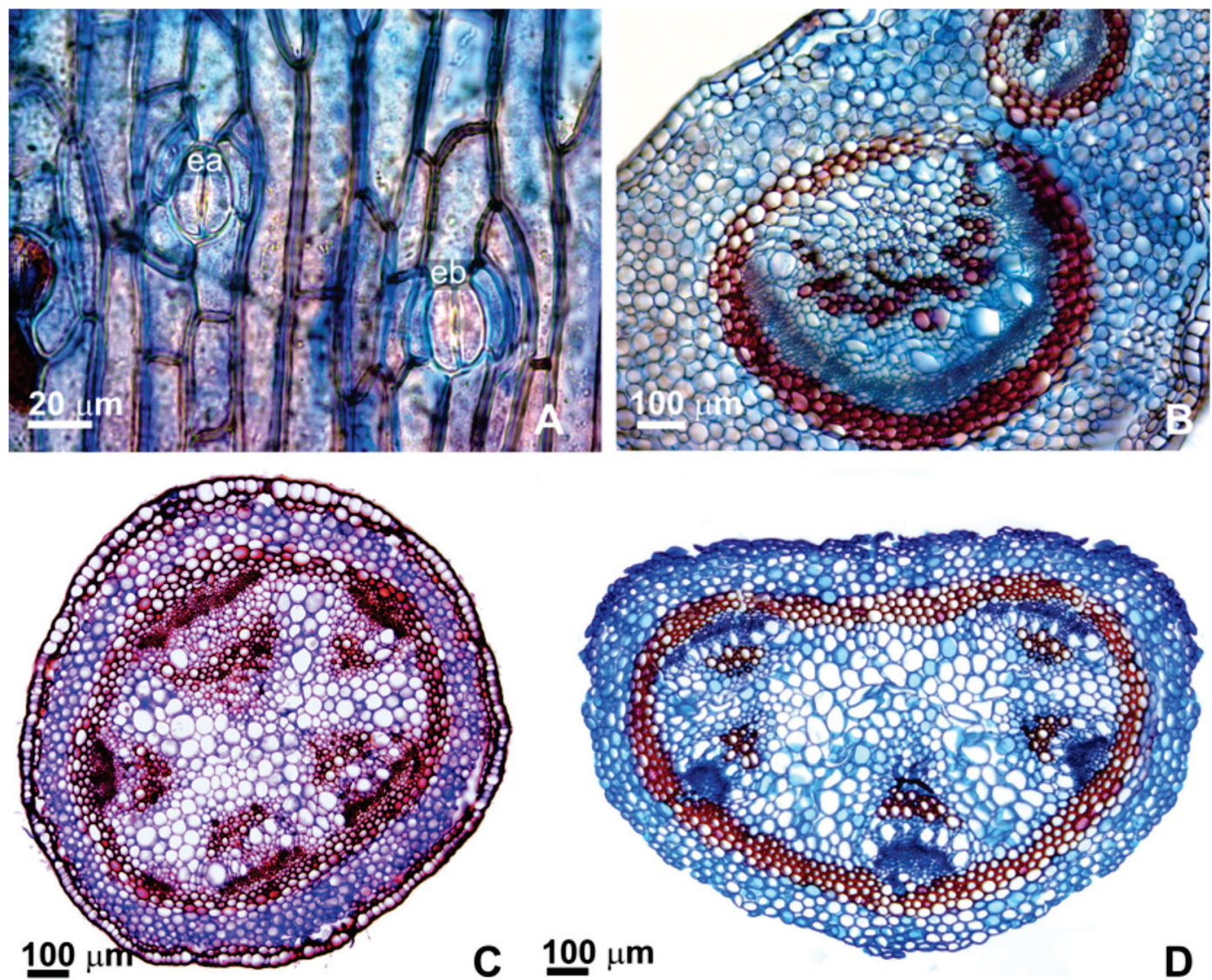

C $100 \mu \mathrm{m}$
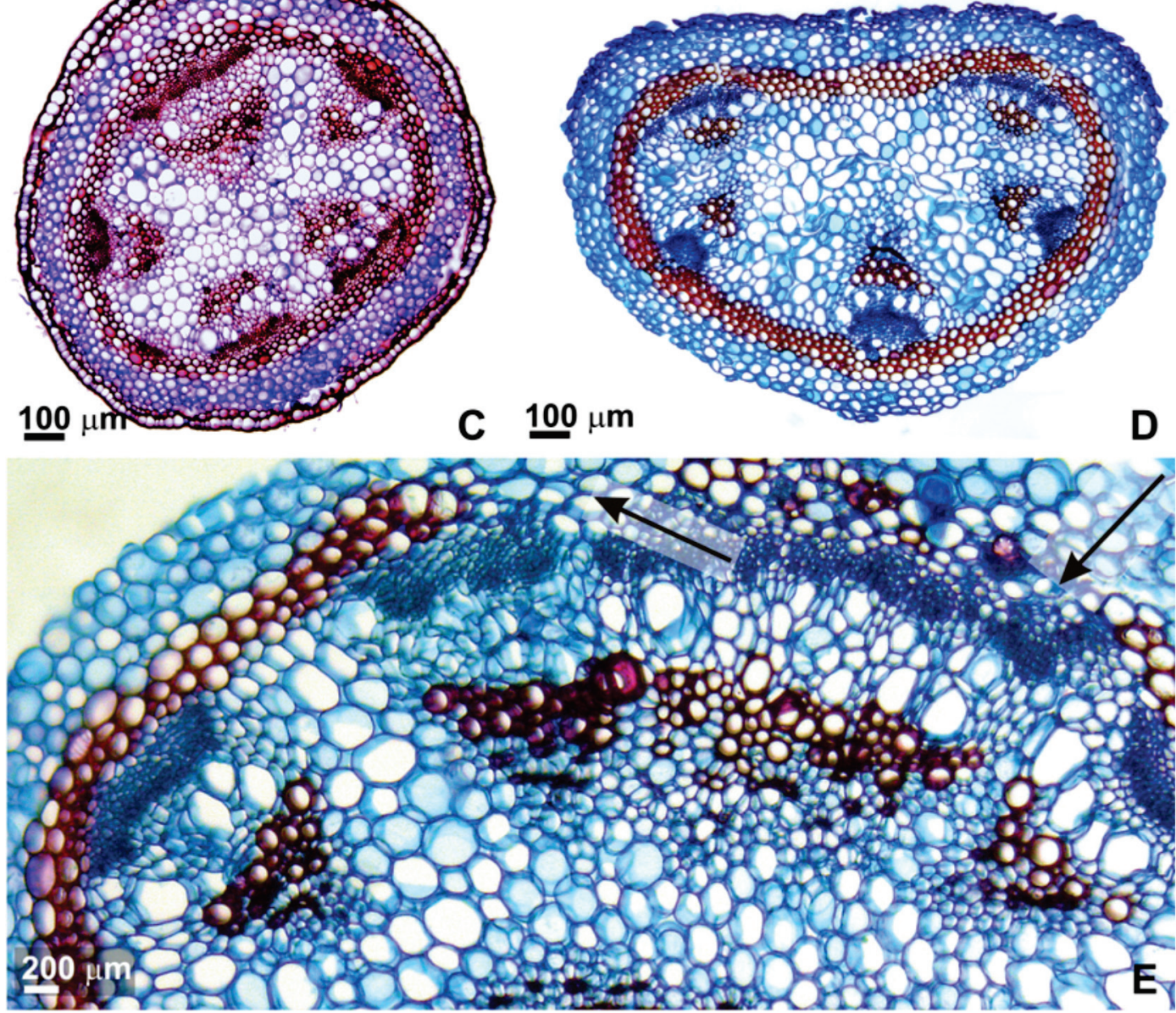

Fig. 5. Tropaeolum incisum, anatomía de pecíolo. A] Vista paradermal. Estomas anomocítico (ea) y braquiparacítico (eb). B) Sección transversal del peciólulo de hoja compuesta. C) Pecíolo de la hoja simple, sección transversal a nivel de la región proximal y media. D) Pecíolo hoja simple, sección transversal a nivel de la región distal. E) Detalle de la sección transversal del pecíolo voluble. 

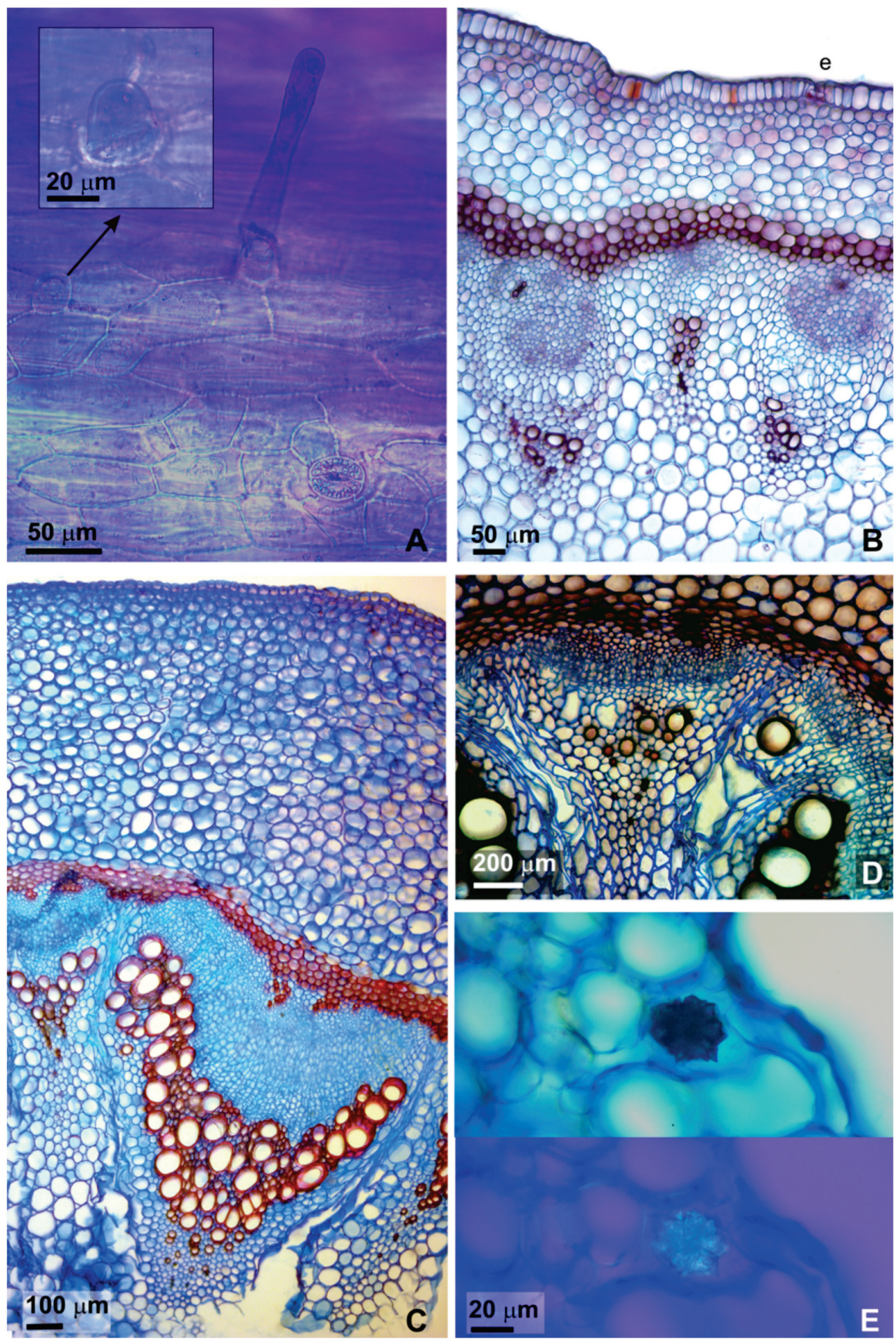

Fig. 6. Tropaeolum incisum, anatomía de tallo. A] Vista paradermal, tallo estructura primaria. Detalle de papila. B] Sección transversal, tallo con estructura primaria. C) Sección transversal, tallo con crecimiento secundario incipiente. D) Colapso de parénquima interfascicular, medular y de los canales secretores esquizógenos ubicados a nivel de cámbium. E) Drusas de oxalato de calcio en médula parenquimática. Referencias: e, estoma. 
pecto rectangular con su eje mayor perpendicular a la superficie, colénquima laminar subepidérmico unistrato y córtex de 4-5 estratos de células parenquimáticas redondeadas. El sistema vascular se halla delimitado por un anillo de 3-4 estratos de fibras esclerenquimáticas y presenta haces vasculares colaterales abiertos con canales secretores esquizógenos a nivel del parénquima xilemático colindante con el cambium vascular, formando una eustela con amplias regiones interfasciculares. La médula presenta células parenquimáticas de mayores dimensiones que las del córtex (Fig. 6 B).

Los tallos maduros de 8-10 mm de diámetro, en vista paradermal, poseen células epidérmicas cuadrangulares de paredes anticlinales rectas, estomas braqui y hemibraquiparacíticos. No se observan tricomas, ni papilas descriptas para el crecimiento primario. En sección transversal se evidencia un estadio de crecimiento secundario incipiente en el cual los haces vasculares individuales se hallan comunicados por cambium interfascicular (Fig. $6 \mathrm{C}$ ). El desarrollo desigual de los diferentes haces y la aparición de xilema secundario causan el colapso del parénquima medular, interfascicular y de los canales secretores esquizógenos del parénquima xilemático (Fig. $6 \mathrm{D})$. La médula se observa parcialmente colapsada con la presencia de drusas de oxalato de calcio (Fig. 6 E).

\section{ANATOMÍA DEL RIZOMA}

Se observa rizoma reservante de aspecto acordonado del cual surgen raíces adventicias de origen exógeno. La epidermis se sustituye tempranamente por una peridermis. En sección transversal presenta peridermis con lenticelas, abundante parénquima cortical amilífero conformado por células cuadrangulares de disposición perpendicular al eje longitudinal del órgano; drusas de oxalato de calcio próximas a la peridermis (Fig. 7 A). El cilindro central es de contorno sinuoso con abundante parénquima medular y radial, el tejido conductor se encuentra formado por haces colaterales abiertos y conjuntos aislados de vasos xilemáticos secundarios (Fig. 7B).

\section{DISCUSIÓN}

Tropaeolum incisum, al igual que otras 7 especies, pertenece a la sección Chilensia. Habita en la estepa, al este y centro oeste de Patagonia, en zonas denominadas por Kalela (1946) como «parque-estepa», puesto que el área de vegetación se encuentra interrumpido por pequeñas isletas de bosques y malezas. Allí precisamente, las plantas deben enfrentar factores ambientales tales como fuertes vientos, períodos prolongados de nieve, alta insolación y en algunos casos salinidad del suelo (Cabrera, 1953).

La suculencia y la ocurrencia de hojas compuestas con folíolos pequeños en $T$. incisum, podría tratarse de una respuesta adaptativa al medio en el que vive ya que este aspecto ha sido señalado como una de las características morfo-anatómicas más notables en plantas que habitan en ambientes que condicionan su crecimiento (Roth, 1992).

El análisis de la anatomía de órganos vegetativos de T. incisum reveló una estructura diferente de las restantes especies de Tropaeolum estudiadas por Metcalfe y Chalk (1950), Fromm-Trinta y Gonçalves-Costa (1976), Zanetti et al. (2004), Bulacio y Ponessa (2012) y Bulacio et al. (2013) quienes señalaron caracteres tales como plantas no suculentas de hojas simples, células con mirosina, mesofilo dorsiventral, papilas en la superficie abaxial, ausencia de tricomas y en algunos casos, de tejido mecánico.

En las hojas tanto simples como compuestas, se observó una epidermis con células poligonales de paredes anticlinales levemente onduladas. Estas características fueron señaladas para las especies sometidas a una baja radiación solar o propias de ambientes mesofíticos, en contraste con lo registrado para las especies xeromórficas donde dichas paredes suelen ser rectas y gruesas (Arambarri et al., 2011). Sin embargo, las ondulaciones de las paredes epidérmicas suele ser un carácter variable por lo que en taxonomía debe ser usado con mesura (Stace, 1965).

La presencia de varios tipos de estomas (anomocíticos, braquiparacíticos, hemipara- 

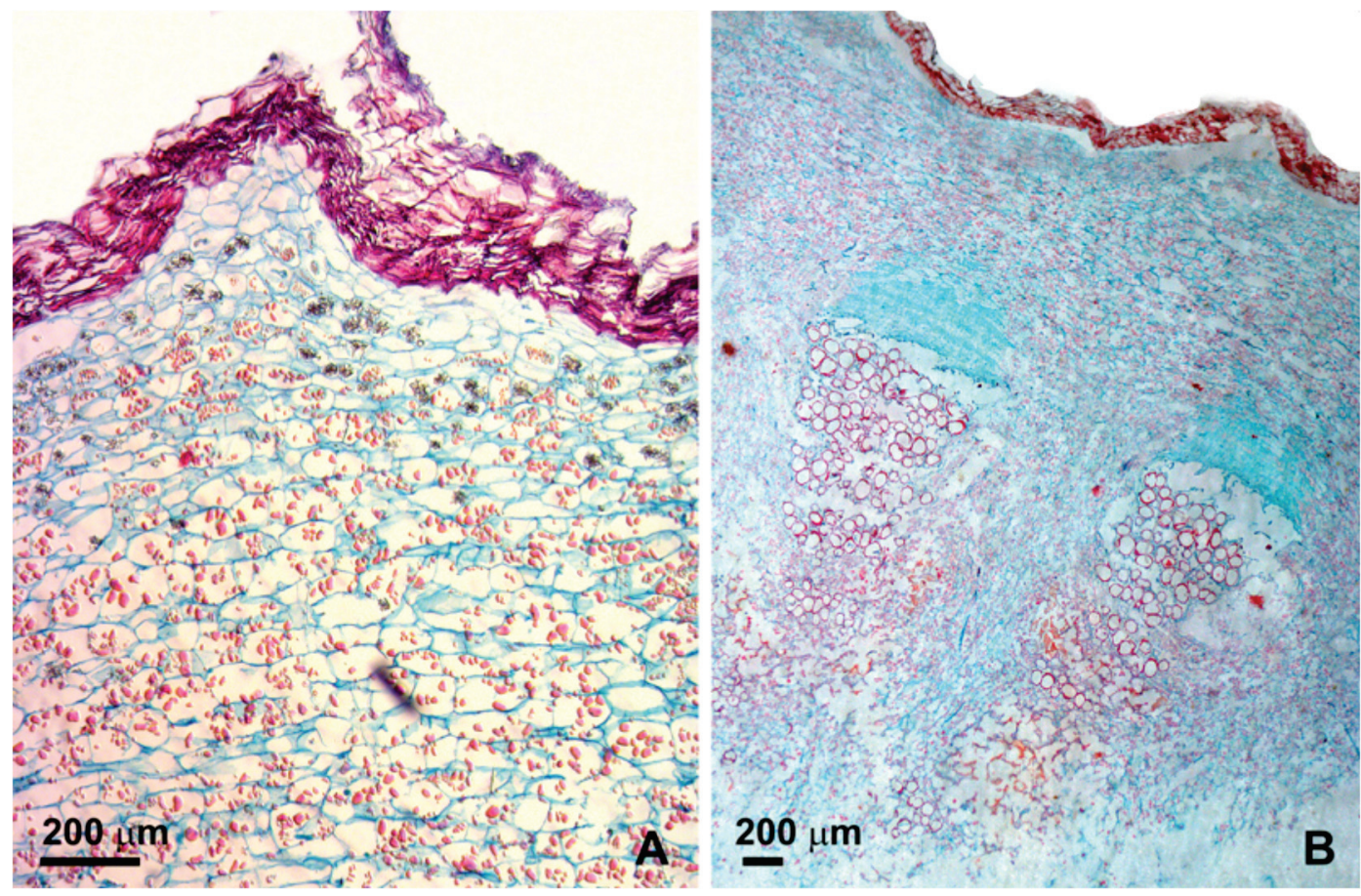

Fig. 7. Tropaeolum incisum, transcorte de rizoma. A] Peridermis con lenticelas y parénquima cortical amilífero. B] Sistema vascular con vasos xilemáticos secundarios aislados, parénquima medular y radial amilífero.

cíticos y raramente estomas gemelos) en una misma hoja es un carácter no registrado para ninguna de las especies de Tropaeolum hasta ahora estudiadas, ya que sólo fueron reportados estomas anomocíticos para la familia (Metcalfe y Chalk, 1950; Fromm-Trinta y Gonçalves-Costa, 1976; Zanetti et al., 2004; Bulacio y Ponessa, 2012 y Bulacio y Ponessa, 2013). Sin embargo, esta variabilidad parece ser bastante común en miembros de otras familias como las Solanáceas (Liscovsky y Cosa, 2005; Palchetti et al., 2014). Por su parte, el mayor tamaño de las células y el mayor número de estomas en la superficie adaxial, reportados en este estudio podría deberse a que la hoja anfiestomática, se pliega exponiendo más la superficie abaxial. En relación a ello, algunos autores señalaron que la disminución en la densidad incrementa la resistencia estomática evitando el exceso de transpiración (Thakur, 1990; Toral et al., 1990). De igual modo Metcalfe y Chalk (1950) citan que plantas con estomas grandes tienden a menudo a reducir su densidad.
Un comportamiento similar se observa en especies patagónicas del género Mulinum Pers. (Apiaceae) (Forcone y Ayestarán, 1996).

Los tricomas además de producir compuestos útiles en la defensa frente a herbívoros y patógenos (Wagner, 1991), tienen importancia en la economía del agua ya que contribuyen a disminuir el aire en toda la superficie que rodea la epidermis, permitiendo así concentrar el vapor de agua y evitar la excesiva transpiración (Fahn y Cutler, 1992), sin embargo la presencia de papilas y tricomas observados en la estructura primaria del tallo, no fueron registradas en tallos maduros ni en las hojas.

Algunos autores mencionaron las estrategias, que tienen algunas plantas, a los ambientes xerofíticos relacionadas con el aumento del espesor de la lámina, microfilia y ciertos cambios anatómicos (Pyykkö, 1966; Dickinson, 2000). La presencia de hojas reducidas en $T$. incisum ya mencionadas por Pyykkö (1966) se complementa con las características anatómicas descriptas en este 
estudio y también morfológicas como ser la presencia de hojas simples y compuestas. Asimismo la ocurrencia de cristales romboidales y drusas de oxalato de calcio en T. incisum, ya fue considerada de valor adaptativo a condiciones áridas y semi áridas para otras especies (Fahn y Cutler, 1992).

El órgano subterráneo (rizoma tuberoso con raíces adventicias exógenas de la especie en estudio), se contrapone al descripto por Pyykkö (1966) como raíz reservante.

Las adaptaciones, no siempre son evidentes a nivel morfológico según indicaron ciertos autores tales como Cutler (1987) y en consecuencia pueden existir adaptaciones fisiológicas que han permitido la evolución hacia formas vegetales aptas para crecer ante situaciones de déficit hídrico y altas temperaturas (Palchetti et al., 2014).

\section{AGRADECIMIENTOS}

A la Fundación Miguel Lillo por haber subsidiado los viajes de campaña para la recolección del material para su estudio.

\section{BIBLIOGRAFÍA}

Andersson L., Andersson S. 2000. A molecular phylogeny of Tropaeolaceae and its systematic implications. Taxon 49: 721-736.

Arambarri A. M., Novoa M. C., Bayón N. D., Hernández M. P., Colares M. N., Monti C. 2011. Ecoanatomía foliar de árboles y arbustos de los distritos chaqueños occidental y serrano (Argentina). Boletín de la Sociedad Argentina de Botánica 46 (3-4): 251-270.

Brücher $H$. 1989. Useful plants of neotropical origin. Berlin Heidelberg, New York; Springer, $296 \mathrm{pp}$.

Bulacio E. 2015. La familia Tropaeolaceae en Argentina. Estudio morfoanatómico y Taxonómico. Tesis Doctoral. Universidad Nacional de Tucumán, Argentina, [inédito, $230 \mathrm{pp}$.].

Bulacio E., Ponessa G. 2012. Morfología y anatomía de órganos vegetativos de Tropaeolum tuberosum ssp. silvestre (Tropaeolaceae). Lilloa 49 (1-2): 3-16.

Bulacio E., Mercado M. I., Ponessa G. 2013. Morfología y anatomía de órganos vegetativos de Tropaeolum capillare [Tropaeolaceae). Lilloa 50 (2): 50-57.
Cabrera A. L. 1953. Esquema fitogeográfico de la República Argentina. Revista del Museo de La Plata Nueva Serie, Botánica 8: 87-168.

Cutler D. F. 1987. Anatomía vegetal aplicada. Librería Agropecuaria. Buenos Aires. $210 \mathrm{pp}$.

Dickinson W. 2000. Integrative plant anatomy. Academic Press, Massachusetts, $533 \mathrm{pp}$.

Dilcher D. L. 1974. Approaches to the identification of Angiosperm leaves. The Botanical Review 40 (1): 1-157.

Dizzeo de Stritmatter C. G. 1973. Nueva técnica de diafanización. Boletín de la Sociedad Argentina de Botánica 15 (1): 126-129.

Ellis B. D., Daly C., Hickey L. J., Johnson H. R., Mitchell J. D., Wilff D., Wing S. L. 2009. Manual of leaf architecture. Cornell University press, USA, 190 pp.

Fabbri L., Valla J. 1998. Aspectos de la biología reproductiva de Tropaeolum penthaphyllum (Tropaeolaceae). Darwiniana 36 (1-4): 51-58

Fahn A., Cutler D.F. 1992. Xerophytes. Handbuchder Pflanzenatomie. Gebruder Borntraeger, Berlin.143 pp.

Fernández J. 1973. Sobre la dispersión meridional de Tropaeolum tuberosum Ruiz et. Pav. Boletín de la Sociedad Argentina de Botánica 15: 106-112.

Forcone A. E., Ayestarán M. G. 1996. Anatomía foliar de cinco especies patagónicas de Mulinum (Apiaceae). Darwiniana 34 (1-4): 121-132.

Fromm-Trinta E., Gonçalves-Costa C. 1976. Tropaeolaceae do Brasil. Bradea 2 (20): 121-148.

Gasparotto Junior A., Boffo M. A., Botelho Lourenço E. L., Alves Stefanello M. E., Leite Kassuya C. A., Andrade Marques M. C. 2009. Natriuretic and diuretic effects of Tropaeolum majus (Tropaeolaceae) in rats. Journal of Ethnopharmacology 22 (3): 517-522.

Hermann M. 1992. Andean roots and tubers: research priorities for a neglected food resource. International Potato Center (CIP): 20-24, Lima, Peru.

Hickey L. J. 1974. Clasificación de la arquitectura de las hojas de Dicotiledóneas. Boletín de la Sociedad Argentina de Botánica 16 (1-2): 1-26.

Kalela A. 1946. Über die steppen und wüstenvetation in Patagonien. Sitzungsberichte Der Finnisch Akademie der Wissenschaften. 1945: 161-172.

Liscovsky I. J., Cosa M. T. 2005. Anatomía comparativa de hoja y tallo en los rep- 
resentantes de Cestreae G. Don (Solanaceae) de Argentina. Gayana Botánica 62: 33-43.

Metcalfe C. R., Chalk L. 1950. Anatomy of the Dicotyledons. Vol. I. Clarendon Press, Oxford, $724 \mathrm{pp}$.

Oppenheimer H. R. 1960. Adaptation to drought: xerophytism. UNESCO: Arid Zone Research15: 105-138.

Palchetti M. V., Barboza G. E., Cosa M. T. 2014. Anatomía foliar en especies de Capsicum (Solanaceae) de diferentes ambientes biogeográficos sudamericanos. Boletín de la Sociedad Argentina de Botánica 49 (3): 417-436.

Pyykkö M. 1966. The leaf anatomy of east Patagonian xeromorphic plants. Annales Botanici Fennicci 3: 453-622.

Roth I. 1992. Leaf structure: coastal vegetation and mangroves of Venezuela. Gebrüder Borntraeger, Berlin. $172 \mathrm{pp}$.

Stace C. A. 1965. Cuticular studies as an aid to plant taxonomy. Bulletin of the British Museum (Natural History), Botany 4: 1-78.
Thakur P. S. 1990. Different physiological responses of tomato (Lycopersicon esculentum Mill.) cultivars to drougth. Acta Physiologiae Plantarum 12: 175-182.

Toral M., Manriquez A., Navarro-Cerrillo R., Tersi D., Naulin P. 1990. Características de los estomas, densidad e indice estomático en secuoya (Sequoia sempervirens] y su variación en diferentes plantaciones de Chile. Bosque 31: 157164.

Wagner G. J. 1991. Secreting glandular trichomes: more than just hairs. Plant physiology 96: 675-679.

Zanetti D. G., Palermo Manfron M., Silva Hoelzel S.C. 2004. Análise morfoanatómica de Tropaeolum majus L. (Tropaeolaceae). Iheringia. Série. Botanica, Porto Alegre 59 [2]: 173-178.

Zarlavsky G. E. 2014. Histología vegetal: Técnicas simples y complejas. Sociedad Argentina de Botánica. Buenos Aires, Argentina, $198 \mathrm{pp}$. 